\title{
Materias primas en las pastas cerámicas de origen volcánico en la cerámica canaria: descripción y caracterización geotécnica
}

\section{Carmen Gloria Martín Afonso a y Fátima Felisa Acosta Hernández}

${ }^{a}$ Facultad de Bellas Artes, Universidad de La Laguna, Calle Radioaficionados s/n, 38200, La Laguna. cgloriamaaf@gmail.com y

${ }^{b}$ Facultad de Bellas Artes, Universidad de La Laguna, Calle Radioaficionados s/n, 38200, La Laguna. ffacosta@ull.edu.es.

\begin{abstract}
Resumen
La tejería de producción semiartesanal es un oficio que está prácticamente desaparecido en el archipiélago canario, y la producción de loza es conservada por unos pocos alfareros/as que han mostrado su interés particular por mantener el oficio, siendo sus piezas objetos decorativos o souvenirs alejándose del uso doméstico que antes cumplían.

La generación actual emplea una terminología referente a las materias primas muy similar, independientemente de la isla donde trabajen. Esto es debido a que consultan las fuentes bibliográficas sobre "las últimas loceras" del Archipiélago, a la mayor comunicación entre loceros/as gracias al contacto en ferias de artesanía insulares, regionales... así como en jornadas de divulgación organizadas por instituciones. No obstante, cuando la cerámica era el medio de vida en los determinados focos alfareros del Archipiélago las loceras denominaban a los materiales según los vocablos locales que les transmitían sus antecesoras, y no siempre un mismo término identificaba a los mismos materiales.
\end{abstract}

En este trabajo se recopilan las referencias etnográficas sobre las materias primas aportando una visión de conjunto y comparativa de las mismas a la vez que se intentan describir aportando algunos datos de carácter más geotécnico que facilite el entendimiento sobre su comportamiento en los procesos cerámicos.

Palabras clave: cerámica, barro, Canarias, volcánico, materias primas, granulometría, índice de plasticidad.

\begin{abstract}
The semi-artisanal weaving factory is a trade that has practically disappeared in the Canary Islands, and the production of earthenware is being conserved by a few potters who have shown their particular interest in maintaining the craft, and now their pieces are just decorative objects or souvenirs, far from the domestic use they used to have.

The current generation uses a very similar terminology for raw materials, regardless of the island where they work. This is due to the fact that they consult the bibliographic sources about the last loceras of the Archipelago, to the greater communication between loceros/as because of the contact between themselves in insular handcraft fairs, or regional ones... as well as in promotion congresses organized by institutions. However, when ceramics were a livelihood in particular potters' centres of the Archipelago, the loceras called the materials according to the local terms transmitted to them by their predecessors, and the same term not always identified equal characteristics materials.

In this work, ethnographic references on raw materials are collected, providing an overview and comparison of them, while an attempt of description of them has been tried by providing some more geotechnical data to facilitate the understanding of their behaviour in the ceramic processes.
\end{abstract}

Keywords: ceramic, clay, Canary Islands, volcanic, raw materials, granulometry, plasticity index. 


\section{Introducción}

A lo largo del siglo XX los oficios tradicionales de Canarias estaban desapareciendo por lo que en torno a la década de los ochenta se desarrollaron abundantes trabajos de investigación sobre ellos, siendo la cerámica un tema que dispone de bastantes publicaciones de diversa índole. Estos trabajos abarcan principalmente estudios etnográficos enfocados a la conservación y recuperación de todos los procesos cerámicos relacionados con la producción de cerámica tradicional y la fabricación de tejas, así como de la vida de las últimas loceras y tejeros.

Cuando aún las loceras sentían que tenían competencia en la producción y venta de su loza mostraban cierto recelo a la hora de informar a los investigadores sobre los lugares de extracción de las materias primas y las proporciones que incorporaban de cada una de ellas cuando elaboraban las pastas. Muestra de ello queda reflejado en las palabras de Rafael González:

(En La Atalaya) "el lugar de obtención del barro es secreto y no nos fue comunicado por las alfareras consultadas. Siempre que hacen referencia a él dicen que lo traen de los secados.” (González, 1977, p. 81)

(En Hoya de Pineda) "el barro lo traen de un lugar cercano, pero tampoco nos fue comunicada su localización, como ocurriera en la Atalaya.” (González, 1977, pp. 84-85)

Pero cuando empezaron a percibir que la demanda de sus piezas era muy pobre y que sus decendientes no continuarían con el oficio mostraron mayor disponibilidad para facilitar estos datos. Es por ello, que en publicaciones posteriores sí podemos encontrar esta información, pero resulta a menudo escasa o incompleta. No obstante, estos datos que se van recopilando de la bibliografía y la hemeroteca facilitaron el inicio de la tesis Estudio y análisis de los barros de origen volcánico en las Islas Canarias. Aplicación y adaptación a la creación en la escultura cerámica (Martín, 2017). y cuyos resultados parciales se recogen en esta comunicación.

\section{Pastas cerámicas}

Una pasta cerámica está formada principalmente por dos componentes: arcilla o materia plástica que se incorpora en mayor proporción y antiplásticos que disminuyen la plasticidad de la arcilla y que ejercen una acción fundente o refractaria en función de la cantidad y la granulometría en la que se añada y la temperatura a la que se cueza la pieza.

\subsection{Pastas comerciales y "tipo"}

Las pastas comerciales o las "tipo" que podemos elaborar siguiendo recetas de un manual emplean materias primas con un alto grado de pureza, cuyas características físico-químicas garantizan el comportamiento de las pastas durante los procesos de construcción, secado y cocción de las piezas. Es por ello, que cuando se compra o elabora una pasta propia se eligen los componentes en función de los objetivos que se persigan diferenciando entre pastas de baja temperatura, pastas de talco, pastas refractarias, pastas de alta temperatura, porcelanas, pastas para moldes...

\subsection{Pastas cerámicas con materias primas de Canarias}

Cuando se desea elaborar una pasta con materiales del entorno, de los que no se dispone información sobre sus cualidades geotécnicas, las guías para ceramistas advierten que "si se utilizan barros locales, se habrá de probar y ajustar convenientemente hasta que den buenos resultados. Se deberá disponer de tres tipos de arcillas de buena calidad, puras y muy plásticas" (Fernández, 1971, p. 86). Esta referencia a la calidad y pureza está vinculada a la escasa presencia de óxidos que actúan como fundentes y colorantes de las pastas, por lo que en términos generales las materias primas de Canarias quedan descartadas para la preparación de recetas "tipo" ya que la geología predominante del Archipiélago está constituida por rocas básicas que al meteorizar en arcillas éstas contienen más de un $20 \%$ de óxidos de hierro y manganeso, y las que proceden de rocas intermedias y sálicas siguen conteniendo estos óxidos en porcentajes del 9-6\%. 


\section{Materias primas de Canarias definidas popularmente}

Los dos grandes componentes de una pasta, tal y como se ha mencionado anteriormente, son la arcilla y los materiales antiplásticos. Dentro de cada grupo, los ceramistas identifican los tipos de arcilla y de antiplásticos a través de nombres internacionalizados y que suelen ser explicados en los manuales como el caolín o china clay, la arcilla de bola, el barro refractario, ... entre los tipos de arcilla; y el feldespato, la sílice o cuarzo,... entre los materiales antiplásticos. Pero las materias primas empleadas en la cerámica canaria no responden a ninguno de estos términos y, en ocasiones, un mismo material recibe diferentes nombres según la Iisla en la que se emplee.

\subsection{Materiales plásticos}

Los materiales con propiedades plásticas eran definidos bajo los siguientes términos:

- Masapé: en el ámbito de la cerámica canaria se le denomina generalmente como barro fuerte, pues se encuentra en el terreno formando agregados muy compactos y con cierto aspecto brillante que resultan muy difíciles de romper (Fig. 1); no obstante, en el diccionario de toponimia canaria encontramos una definición mucho más amplia:

"La forma más común con que aparece este étimo en la toponimia de Canarias es la de Masapés, pero también se documenta, oralmente, las formas de Masapé y Masapén, y por escrito, Masapez (Gran Canaria) y Mazapez (en La Palma) y sus respectivos derivados Masapeces y Mazapeces. Y por debajo de la toponimia, es palabra común en las hablas insulares de Gran Canaria, Tenerife, La Palma y El Hierro para referir a un tipo de tierra. Sin embargo, la referencia al tipo de tierra no es igual en todas partes. Ya en portugués, de donde masapés procede, designa varias cosas: 'tierra fértil, como consecuencia de los álcalis que en ella abundan' y 'barrial' (en Brasil y Cabo Verde), y 'tierra volcánica que mezclada con cal sirve como cemento' (en Azores). En Canarias, el masapés puede identificarse, según las Islas, con la arcilla, con el barro que se usa en la alfarería tradicional, con un barrizal, con un terreno improductivo y, en El Hierro, con una tierra arenisca, muy fina y rojiza, totalmente impermeable" (Trapero, 1999, p. 278).

- Barro flojo: "El barro flojo se diferencia del fuerte en que sus partículas son de mayor tamaño y por consiguiente resulta menos plástico. Se encuentra normalmente en los terrenos de cultivo, en forma de terrones opacos que se deshacen fácilmente, sobre todo secos, y pueden reducirse a polvo fácilmente (Fig. 2). Tradicionalmente se ha usado en la elaboración de tejas y ladrillos, y puede formar parte de la mezcla como desgrasante para evitar el exceso de arena." (Afonso, 1983, p. 26).

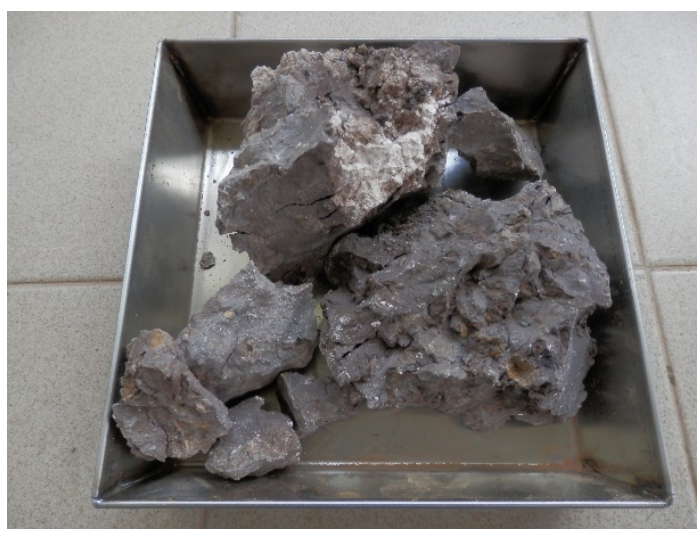

Fig. 1 Barro fuerte procedente de Haría, Lanzarote

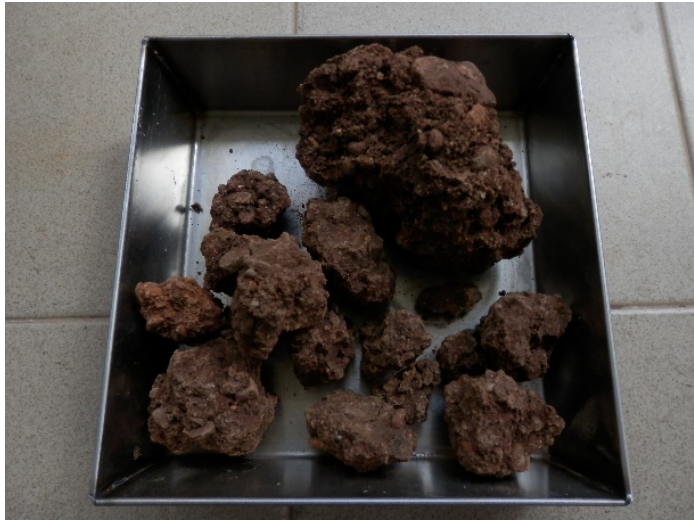

Fig. 2 Barro flojo procedente de Jeva, Tenerife

- Barro terrento: El término terrento es poco frecuente y apenas aparece en las fuentes bibliográficas. Únicamente se menciona cuando se hace alusión a la cerámica de La Victoria de Acentejo en Tenerife y a dos 
de sus últimas alfareras: Dña. Adela Hernández González, conocida como Adela Brito (Fernández, 2002, pp. 222-223) y Dña. Fidelina Gutiérrez Pérez. No obstante, en unas menciones se le asemeja a un barro flojo:

"El método utilizado por Dña. Adela en otro tiempo ofrecía unas características muy especiales que merece la pena analizar. El barro fuerte, semejante al mazapé o greda era el que deba más plasticidad a la masa. Para evitar los inconvenientes que presentaba se le añadía barro terrento para aflojarlo y desgrasarlo. El arenuco, que, según sus explicaciones, era un barro que contenía arena, actuaba como desgrasante." (Afonso, 1983, p. 28)

"Cogíamos tres tipos de barro, fuerte, terrento y arenuco. Cada uno tenía un sitio, el terrento estaba más arriba, el fuerte abajo, y del arenuco se ponían menos porque era para aflojar el fuerte y el terrento. Todos los barros estaban en la misma finca, pero más abajo, más arriba... [...] Yo sabía la diferensia de los barros porque trabajé desde chiquitita. El barro fuerte lo ve usted al aire, en la tierra de arriba, agarra usted con la asada y lo coge de arriba. El que es más terrento costaba más pa cavar y el arenuco que está más pabajo. Yo misma cavaba, tenía fuersas pa es.” (González, 1977, pp. 34-35)

$\mathrm{Y}$ en otras se le identifica con un barro fuerte:

"Fidelina Gutiérrez Pérez es una de las últimas alfareras tradicionales de La Victoria de Acentejo. [...] Para trabajar usa un barro terrento (fuerte) y uno arenoso; el fuerte es gris, y el arenoso, medio amarilloso". (Eléxpuru y Martínez, 2000, p. 151).

- Barro arenuco: Este término se localiza en la bibliografía junto al de barro terrento, pues era empleado por las alfareras de La Victoria anteriormente mencionadas. Como explicaban ellas, y su nombre da a entender, contenía de manera natural bastante arena cumpliendo una función de antiplástico, pero por conservar su identificación etnográfica mantenemos su clasificación dentro de las materias plásticas.

- Tierra de teja: Es la que se empleaba para hacer las tejas y cuando se describe se identifica como una tierra fuerte.

"El más empleado era el tipo arcilloso "barro fuerte" o "tierra de teja", reconocido por su textura pegajosa." (Rodríguez, y Rodríguez, 2008, pp. 97-99).

"Casi siempre, el barro se cavaba en las inmediaciones del horno: "lo cogíamos por aquellos redores; no hay mucho piso, cogíamos una poquita en un sitio, otra en otro". [...] Según nos refirió el amasador, D. Manuel Pérez, una parte del barro tenía que ser fuerte y la otra floja, porque si no las tejas podrían "estallarse" en el horno: "eso si se conoce; el barro fuerte es más duro, el flojo es más momio, más flojo"." (Lorenzo, 1987, p. 60).

"Barro y agua son los materiales empleados en la confección de las tejas. Se usaba barro fuerte y flojo, a partes iguales (Teno Alto); o, únicamente, una clase de barro fuerte, tierra de teja (La Hoya)." (Lorenzo, 1998, p. 90).

"Se usaba una clase de barro fuerte conocido como "tierra de teja", al que se reconocía por ser un barro pegajoso, abundante en los terrenos "malos" que no se araban "lo más que se cría en ella es el guargaso (jara)"." (Lorenzo et al., 1993, p. 11).

"El barro lo clasificaban en tres tipos según fuese su utilidad para la tejería: la tierra fuerte, la tierra de cultivo y mazapé. La tierra fuerte era la de mejor calidad, la más resistente y por ello la utilizada para la teja y las losetas. Los treinta o cuarenta centímetros del terreno eran la tierra de cultivo, que una vez limpia se utilizaba para los ladrillos.” (Espinel y Pérez, 1995, p. 33).

"Hay otro lugar [en Temejiraque, El Hierro] donde se ven vestigios de un horno que hubo de teja. Aquella tierra es una tierra fuerte que bajaba desde arriba por las barranqueras que había." (Sánchez, 1998, p. 102). 


\subsection{Materiales antiplásticos}

Los materiales que se añadían a la pasta para reducir la plasticidad eran definidos globalmente como desgrasantes. Los alfareros especificaban dentro de este término genérico los siguientes:

- Arena de barranco: es el antiplástico más mencionado en la bibliografía, pero es un término que sólo hace referencia al lugar de extracción y cuya composición está condicionada por la geología del entorno y zonas más altas del cauce donde se recoja, a lo que se suman los inconvenientes de la redondez del grano y poder de estar contaminada de cal.

- Arena volcánica: También se la define como arena de minas o picón, pero geológicamente se corresponde con piroclastos basálticos denominados lapilli. Éste puede encontrarse en el Archipiélago en torno a los conos volcánicos y puede ser de color rojo o negro, ya que "originalmente, fueron oscuros pero una rápida oxidación, o una lenta meteorización, les confieren un típico color rojizo." (Araña y Carracedo, 1980, p. 163).

- Tosca o toba: La toba procede también de productos piroclásticos, pero que "se depositan en masas caóticas sin estratificación alguna. Estos casos responden generalmente al violento proceso explosivo que sucede a un taponamiento de la boca eruptiva. Es tan alto el volumen de piroclastos producidos al destupirse nuevamente el conducto, que se forma una densa nube, la cual no puede elevarse debido al gran porcentaje de fragmentos rocosos que lleva en suspensión. Se provoca entonces un flujo turbulento de cenizas (ash-flow), que se desplazan con alta velocidad al ras del suelo, hasta que se depositan caótica pero unitariamente, y todavía ardientes, pese al carácter fragmentario del material." (Araña y Carracedo, 1978, p. 50). Cuando la composición del material eruptivo es sálica, los productos pétreos resultantes adquieren colores claros los cuales sólo aparecen (salvo excepciones) en las islas de Tenerife y Gran Canaria debido a que proceden del volcanismo de las Etapas Intermedias de formación del Archipiélago.

- Chamota: la chamota sí es un término y material conocido entre los ceramistas, pero es importante diferenciar entre:

"Chamota de caolín calcinado: también conocida como "chamota Peser". Procede de la calcinación de caolín pétreo, constituido principalmente por caolinita. Debido a su previa calcinación, aporta elevada estabilidad a la vez que rebaja la plasticidad." (Garraza, 1992-93, p. 48)

"Chamota de recuperación: proviene de la molienda de material cerámico ya cocido. Puede presentar problemas por la presencia de caliches, por lo que aconsejamos usarla en granulometrías bajas $(0-0,5)$. Llega a alterarse al entrar en contacto con ciertos materiales fusibles." (Garraza, 1992-93, p. 50)

En la cerámica canaria se empleaba la chamota de recuperación como material de reaprovechamiento cuando salía alguna pieza rota del horno.

- Conchas: son un desgrasante que aporta carbonato cálcico y que se ha detectado en análisis de piezas prehispánicas. En la cerámica que se produce actualmente no se emplea sino en casos puntuales donde se quieren elaborar reproducciones de piezas aborígenes intentando emular en la mayor medida de lo posible el proceso de fabricación de los antiguos pobladores de las Iislas.

- Levaduras o raspas de mestura: son "las esquirlas de barro residuales en el raspado de las vasijas anteriores" (Lezcano, 1944, pp. 176-177). Estos recortes de trabajos previos suponen un material de aprovechamiento y reutilización en el alfar que permiten disponer de mayor cantidad de pasta para modelar sin necesidad de recurrir a materias primas y de aportar mayor plasticidad a la masa porque "existen partículas de barro muy graso o fuerte que no se deshacen en un primer amasado, sino tras un largo período de remojo en agua" (Afonso, 1983, p. 46), que favorece la maduración de la arcilla por conservarse en lugares húmedos (Fourier 1981, p. 15, voz Almacenamiento de la arcilla) y porque la proliferación de bacterias favorece la formación de geles ácidos y la descomposición vegetal que contribuyen al intercambio iónico que da lugar a la plasticidad (Fourier, 1981, p. 35, voz Bacterias en la arcilla). 


\section{Características geotécnicas de algunas materias primas}

Los materiales plásticos, la arcilla, son los que están presentes en mayor cantidad en una pasta cerámica por lo que se han seleccionado veinticinco muestras de suelo procedentes de las islas Canarias (Fig. 4) para realizar su caracterización geotécnica e intentar establecer relaciones entre las descripciones populares sobre las materias primas recogidas anteriormente y los resultados obtenidos en los estudios de laboratorio.

Para la extracción de las muestras se partió en primer lugar de la información bibliográfica proporciada en las investigaciones etnográficas y en segundo lugar por alfareros/as que siguen trabajando la cerámica canaria. En algunos casos, los propios artesanos nos acompañaron al lugar de extracción y en última instancia nos servimos de lugareños interesados por la cerámica canaria para localizar las barreras de las que sólo disponíamos unos pocos datos de referencia.

\subsection{Estudios normalizados realizados}

Entre los estudios normalizados a los que se sometieron las muestras se encuentran el análisis granulométrico y los límites de Atterberg.

Con el análisis granulométrico se determina el tamaño y cantidad de las partículas que contiene la muestra. Mediante el tamizado se obtienen los tamaños correspondientes a 125, 100, 80, 63, 50, 40, 25, 20, 12,510, 6'3, 5, 2 y 1'25 mm de diámetro, mientras que para tamaños inferiores se emplea el método del densímetro donde se llega a definir la cantidad de partículas inferiores a $2 \mu$ que son las consideradas arcillas, cuyo dato resulta de mayor interés en este estudio para definir la cantidad de arcilla que contiene la muestra de forma natural.

Con los límites de Atterberg se determinan los porcentajes de agua que debe contener la muestra para que se comporte como un líquido y para que no sea modelable entre las manos. La relación entre estos datos permite definir el Índice de Plasticidad y situar las muestras en el Gráfico de Casagrande el cual está dividido en cuatro secciones principales en las que se distinguen: CL (clay low) correspondiente a arcillas con Límite Líquido inferior a 50 y CH (clay high) a arcillas con Límite Líquido superior a 50. ML y MH corresponden a limos inorgánicos (M es la inicial de mo, palabra sueca), y $\mathrm{OL}$ y OH corresponden a suelos con materia orgánica coloidal.

\subsection{Datos obtenidos}

La realización de los estudios nos proporciona una serie de datos que se han organizado conjuntamente en la tabla 1, la cual está dividida en tres bloques:

- En el primero, se definen las veinticinco muestras estudias indicando la isla de procedencia, su numeración en el mapa y la abreviatura empleada para situarlos en determinados gráficos.

- En el segundo, qué referencias se han tenido en cuenta para extraer dicha muestra. Se puede observar cómo en algunas muestras se parte de la bibliografía y posteriormente nos vemos respaldadas por la información directa del contacto con un alfarero/a que incluso nos acompaña a la barrera, mientras que en otras sólo disponemos de información bibliográfica y de algún lugareño.

- En el tercero, se registran los porcentajes obtenidos de cantidad de partículas inferiores a $2 \mu$, y los porcentajes de agua que definen el Límite Líquido y el Límite Plástico, así como la relación entre estos dos, Índice de Plasticidad.

Las muestras de suelo estudiadas contienen porcentajes de partículas inferiores a $2 \mu$ muy variados:

- Menos de 45\%: Las Albercas 1, Las Albercas 2, Puntallana, Chirche, Jeva, Tejina, Lugarejos, Morro Velosa, Vallebrón, Las Nieves.

- Entre 45 y 55\%: Arguamul, El Cercado, Tunte, La Atalaya.

- Entre 55 y 66\%:Valverde, Santa Brígida, Haría.

- Entre 66 y 76\%: Putnagorda, El Tanquillo, El Púlpito, Riquiánez, Los Soldados 1, Los Soldados 2.

- Más de 76\%: Arguayo, Hoya de Pineda. 
Tabla 1. Referencias para la extracción de las muestras y datos obtenidos en los estudios

\begin{tabular}{|c|c|c|c|c|c|c|c|c|c|c|c|}
\hline \multirow[b]{2}{*}{ 离 } & \multirow[b]{2}{*}{ 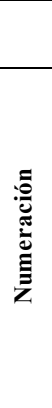 } & \multirow[b]{2}{*}{ 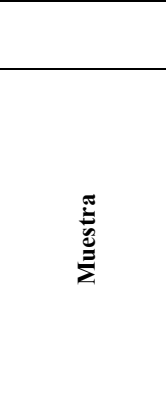 } & \multirow[b]{2}{*}{ 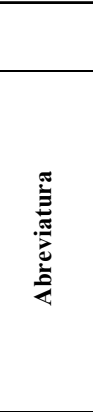 } & \multicolumn{4}{|c|}{$\begin{array}{l}\text { Referencias para la } \\
\text { localización y extracción }\end{array}$} & \multicolumn{4}{|c|}{ Estudios normalizados UNE } \\
\hline & & & & 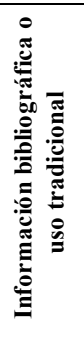 & 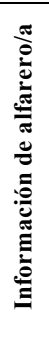 & 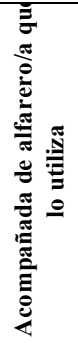 & 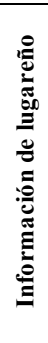 & 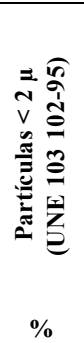 & 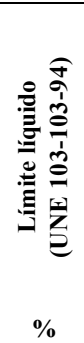 & 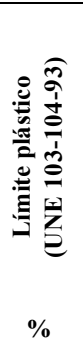 & 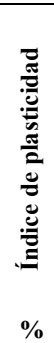 \\
\hline \multirow{3}{*}{ El Hierro } & 1 & Las Albercas 1 & AL1 & & & & $\mathrm{X}$ & 29,3 & 39,2 & 36,9 & 2,3 \\
\hline & 2 & Las Albercas 2 & AL2 & $\mathrm{X}$ & $\mathrm{X}$ & & & 43,8 & 76,4 & 43,4 & 32,9 \\
\hline & 3 & Valverde & VAL & $\mathrm{X}$ & $\mathrm{X}$ & & & 61,5 & 77,1 & 36,8 & 40,4 \\
\hline \multirow{2}{*}{ La Palma } & 4 & Puntagorda & PG & $\mathrm{X}$ & $\mathrm{X}$ & & $\mathrm{X}$ & 73,3 & 80,3 & 42,1 & 38,2 \\
\hline & 5 & Puntallana & PLL & $\mathrm{X}$ & & & $\mathrm{X}$ & 28,4 & 63,2 & 34,4 & 28,9 \\
\hline \multirow{2}{*}{ La Gomera } & 6 & Arguamul & MUL & & $\mathrm{X}$ & & & 53,7 & 94,8 & 32,6 & 62,2 \\
\hline & 7 & El Cercado & CER & $\mathrm{X}$ & $\mathrm{X}$ & $\mathrm{X}$ & & 51,5 & 71 & 37,3 & 33,6 \\
\hline \multirow{6}{*}{ Tenerife } & 8 & Arguayo & AYO & $\mathrm{X}$ & $\mathrm{X}$ & $\mathrm{X}$ & & 79,9 & 92,8 & 26,4 & 66,4 \\
\hline & 9 & Chirche & $\mathrm{CHI}$ & $\mathrm{X}$ & & & $\mathrm{X}$ & 11,6 & 25,7 & 16,7 & 8,9 \\
\hline & 10 & El Tanquillo & TAN & $\mathrm{X}$ & $\mathrm{X}$ & $\mathrm{X}$ & & 69,8 & 67,2 & 33,9 & 33,4 \\
\hline & 11 & Jeva & JEV & $\mathrm{X}$ & $\mathrm{X}$ & $\mathrm{X}$ & & 25,6 & 35,7 & 23,1 & 12,6 \\
\hline & 12 & Tejina & TEJ & & $\mathrm{X}$ & & $\mathrm{X}$ & 43,5 & 75,5 & 35,4 & 40,2 \\
\hline & 13 & El Púlpito & PUL & & $\mathrm{X}$ & $\mathrm{X}$ & & 72,9 & 71,8 & 35,4 & 36,9 \\
\hline \multirow{6}{*}{ Gran Canaria } & 14 & Lugarejos & LUG & $\mathrm{X}$ & $\mathrm{X}$ & & & 40,5 & 59,7 & 31,2 & 28,4 \\
\hline & 15 & Hoya de Pineda & HOP & $\mathrm{X}$ & & & $\mathrm{X}$ & 78,9 & 63,4 & 31,8 & 31,6 \\
\hline & 16 & Tunte & TUN & $\mathrm{X}$ & $\mathrm{X}$ & $\mathrm{X}$ & & 52,9 & 62,1 & 30,3 & 31,8 \\
\hline & 17 & Riquiánez & RI & $\mathrm{X}$ & & & $\mathrm{x}$ & 72,9 & 76,6 & 38,2 & 38,4 \\
\hline & 18 & Santa Brígida & STB & $\mathrm{x}$ & $\mathrm{x}$ & & & 64,8 & 69,7 & 32,1 & 37,7 \\
\hline & 19 & La Atalaya & ATA & $\mathrm{X}$ & $\mathrm{X}$ & $\mathrm{X}$ & & 45,7 & 51,5 & 22,9 & 28,5 \\
\hline \multirow{2}{*}{ Fuerteventura } & 20 & Morro Velosa & MV & $\mathrm{X}$ & $\mathrm{X}$ & & & 26,7 & 59,7 & 37,6 & 22,2 \\
\hline & 21 & Vallebrón & BRON & & $\mathrm{X}$ & $\mathrm{X}$ & & 27,7 & 59,3 & 36,3 & 22,8 \\
\hline \multirow{4}{*}{ Lanzarote } & 22 & Las Nieves & NIE & $\mathrm{X}$ & $\mathrm{X}$ & $\mathrm{X}$ & & 39 & 77,9 & 45 & 32,9 \\
\hline & 23 & Los Soldados 1 & SO1 & & $\mathrm{X}$ & $\mathrm{X}$ & & 75,8 & 94,4 & 36,2 & 58,2 \\
\hline & 24 & Los Soldados 2 & $\mathrm{SO} 2$ & & $\mathrm{X}$ & $\mathrm{X}$ & & 73,4 & 105,5 & 41 & 64,5 \\
\hline & 25 & Haría & HA & & $X$ & X & & 65,9 & 126,9 & 48,6 & 78,3 \\
\hline
\end{tabular}

\section{GRÁFICO DE CASAGRANDE}

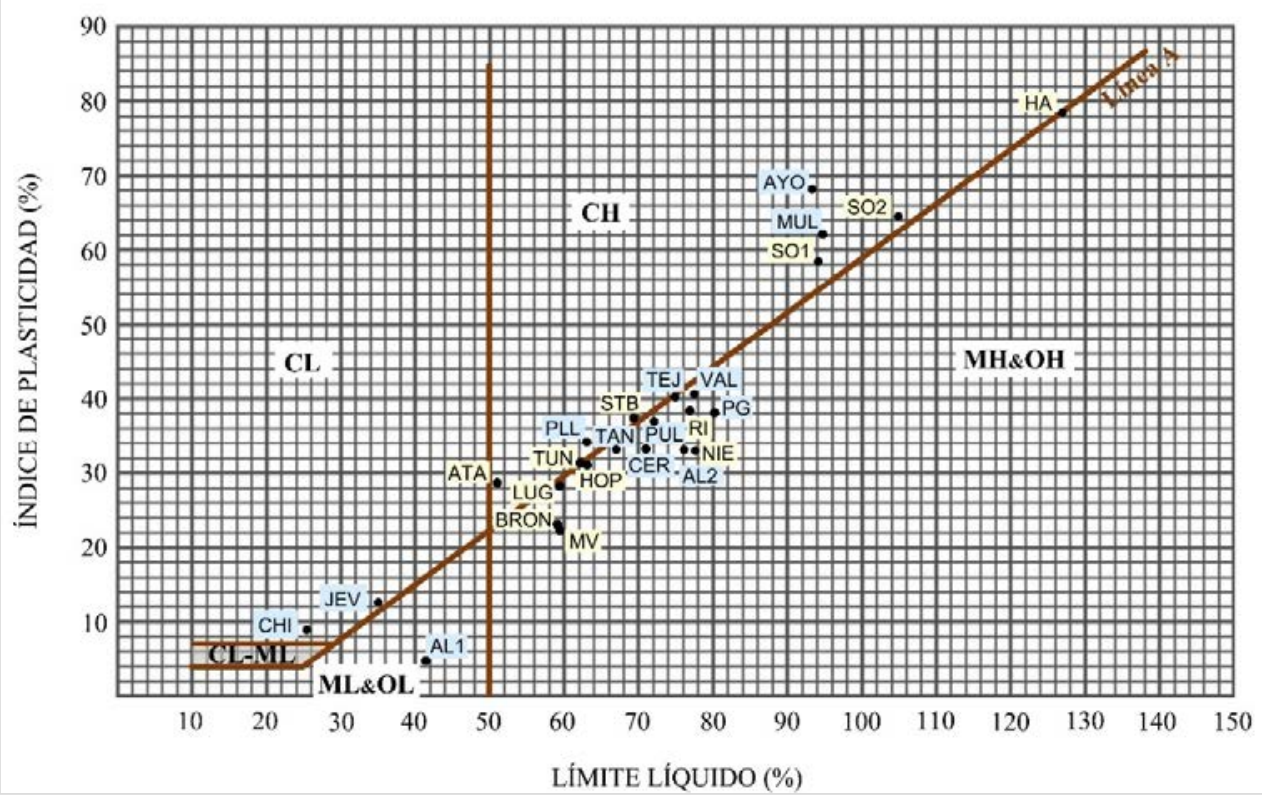

Fig. 3 Muestras situadas en el Gráfico de Casagrande 
En la figura 3, se sitúan las muestras según los datos obtenidos tras la realización del estudio del Límite Líquido y la relación de éste con el Límite Plástico, Índice de Plasticidad. Se observa que las muestras se reparten en tres grupos:

- Las muestras que se tienen un Límite Líquido inferior a 50: Chirche, Jeva y Las Albercas 1.

- Las muestras que tiene su Límite Líquido entre 50 y 80: La Atalaya, Vallebrón, Lugarejos, Morro Velosa, Tunte, Hoya de Pineda, Puntallana, El Tanquillo, El Cercado, Las Albercas 2, El Púlpito, Santan Brígida, Tejina, Las Nieves, Riquiánez, Valverde y Puntagorda.

- Las muestras que tienen un Límite Líquido superior a 90: Los Soldados 1, Arguamul, Arguayo, Los Soldados 2 y Haría.

\section{Conclusiones}

La investigación realizada sobre los barros de Canarias nos ha proporcionado observaciones significativas sobre la especificidad del lenguaje en relación con la manipulación propia de las muestras de suelo extraídas para su estudio en el laboratorio y los datos técnicos obtenidos. De esta interrelación se destacan las conclusiones más significativas.

La comparación de los datos obtenidos en los estudios realizados, porcentaje de partículas inferiores a $2 \mu$ e Índice de Plasticidad reflejada en la figura 4, nos conduce a la conclusión inicial de que no hay una correspondencia regular que indique que a mayor cantidad de partículas arcillosas la muestra tenga mayor Índice de Plasticidad, tal y como se muestra en el gráfico comparativo.

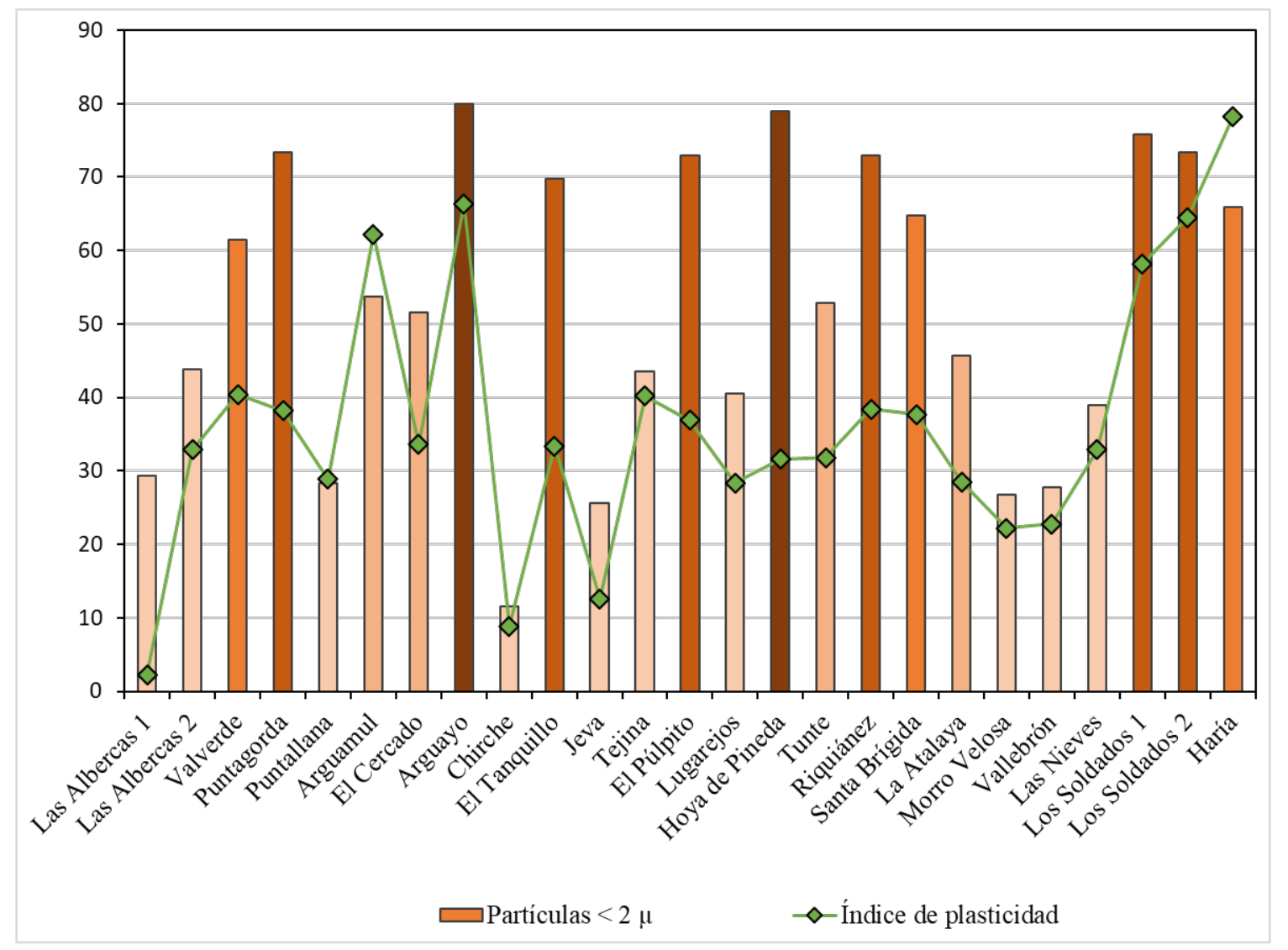

Fig. 4 Comparación gráfica de los datos obtenidos en porcentaje de partículas inferiores a $2 \mu$ e Índice de plasticidad

No obstante, a partir del Gráfico de Casagrande se puede determinar que las muestras con Límite Líquido inferior a 50 serían definidas por los alfareros como barros flojos, mientras que las muestras con Límite Líquido superior a 90 serían definidas por los alfareros como masapé o barros fuertes, que a su vez tienen porcentajes de partículas tipo arcillas superiores al 66\% a excepción de la muestra procedente de Arguamul. 
La mayor complejidad se presenta en el grupo de muestras concentradas en el centro del Gráfico. Todas se encuentran en torno a la Línea A que separa arcillas de suelos con materia orgánica coloidal y con Límite Líquido superior a 50. Si sólo se tuviera en cuenta este gráfico se podrían definir como barros fuertes, pero las descripciones de los artesanos y la manipulación de los agregados en el laboratorio antes de someterlos a estudios nos lleva a otras consideraciones.

Muestras como Vallebrón, Morro Velosa, Hoya de Pineda, Las Albercas 2 y El Cercado se extrajeron a no demasiada profundidad. Sus agregados eran más bien pequeños y poco brillantes por lo que probablemente a ojos de un alfarero/a serían definidos como barros flojos.

La muestra de Las Nieves, se extrajo del lugar empleado tradicionalmente y según las descripciones etnográficas era considerado un barro fuerte. No obstante, al compararlo con el resto de las muestras extraídas de Lanzarote, las cuales fueron posibles gracias a excavaciones mecánicas que dejaban a la vista horizontes del terreno de mayor profundidad, podría considerarse un barro flojo.

Los agregados de las muestras de Tejina, Valverde, Puntagorda, Riquiánez, Tunte, El Púlpito y El Tanquillo sí se correspondían con la descripción de un masapé de caras brillantes y mucha dificultad para deshacerlo con la mano.

Las muestras de Lugarejos, Puntallana y Santa Brígida se encuentran en una posición intermedia difícil de definir por la cantidad de partículas de tamaños superiores que se incorporaron en el momento de la extracción de la barrera.

La muestra de La Atalaya resulta un caso diferenciado cuyas cualidades son apreciadas por todos los alfereros/as del Archipiélago para la producción de cerámica. En La Atalaya nunca se ha diferenciado entre barro fuerte y barro flojo, ya que, tradicionalmente, siempre se había utilizado el mismo barro y como ofrecía tan buenos resultados durante los procesos cerámicos simplemente se le definía como barro.

\section{Referencias}

Afonso García, M. (1983). Greda. Manual de alfarería popular canaria. La Orotava, Tenerife: Asociación cultural Pinolere.

Araña, V., y Carracedo, J. C. (1978). Los volcanes de las Islas Canarias I. Tenerife. Madrid: Editorial Rueda.

Araña, V., y Carracedo, J. C. (1980). Los volcanes de las Islas Canarias III. Gran Canaria. Madrid: Editorial Rueda.

Eléxpuru, I., y Martínez Zafra, J. C. (2000). Guía de Artesanía de Tenerife. Madrid: Consejería de Industria y Comercio, Gobierno de Canarias.

Espinel Cejas, J. M., y Pérez Prieto, M. E. (1995). El aprovechamiento de los recursos del medio en la elaboración tradicional de tejas, ladrillos y losetas (La Laguna, Tenerife). Tenique. Revista de Cultura Popular Canaria, 3, 25-59. Canarias: Grupo Folklórico de la Escuela de Magisterio de La Laguna, patrocina Vicerrectorado de Alumnado de la ULL.

Fernández Chiti, J. (1971). Curso práctico de cerámica (Vol. I). República Argentina: Ediciones Condorhuasi.

Fernández García, A. (2002). La Victoria. 5 siglos. Tenerife: Centro de la Cultura Popular Canaria.

Fourier, R. (1981). Diccionario ilustrado de alfarería práctica. Barcelona: Ediciones Omega.

Garraza Salanueva, A. (1992-93). Experimentación de las propiedades de las pastas cerámicas para una práctica artística. Tesis doctoral. Bilbao: Universidad de País Vasco.

González Antón, R. (1977). La alfarería popular en Canarias. (Ed. 1987.) Con la colaboración de Manuel J. Lorenzo Perera. Santa Cruz de Tenerife: Aula de Cultura de Tenerife, Museo etnográfico, Publicaciones científicas del Excmo. Cabildo Insular de Tenerife.

Lezcano Montalvo, P. (1944). Visita a La Atalaya de Gran Canaria. En Tradiciones populares. Palabras y cosas. Colección de ensayos y notas de folklore canario. Tomo I (pp. 171-184). La Laguna: Consejo Superior de Investigaciones Científicas, Instituto de Estudios Canarios.

Lorenzo Perera, M. J. (1987). Estampas etnográficas de Teno Alto. Madrid: Ayuntamiento de Buena Vista del Norte.

Lorenzo Perera, M. J. (1998). Los estudios sobre la tejería tradicional en las Islas Canarias. Una reflexión y un reto. En IV Congreso Internacional: Rehabilitación del Patrimonio Arquitectónico y Edificación, La Habana, Cuba (pp. 90-93). España: Centro Internacional para la Conservación del Patrimonio. CICOP. 
Lorenzo Perera, M. J. et al. (1993). El horno de tejas de La Hoya (San Miguel de Abona). Trabajos de investigación, limpieza y reconstrucción. Tenique. Revista de Cultura Popular Canaria, 1, 1-32. La Laguna: Grupo Folklórico de la Escuela de Magisterio de La Laguna.

Martín Afonso, C. G. (2017). Estudio y análisis de los barros de origen volcánico en las Islas Canarias. Aplicación y adaptación a la creación en la escultura cerámica. Tesis doctoral. La Laguna: Tesis Doctoral. Universidad. Disponible en http://riull.ull.es/xmlui/handle/915/16201

Rodríguez Adriani, M. C., y Rodríguez Tejera, J. (2008). Restos de una tradición en el olvido. Tipologías y catalogación de Hornos. En Arico. Santa Cruz de Tenerife: Autoedición.

Sánchez Perera, S. (1998). La producción de teja en la Isla de El Hierro. El Pajar. Cuaderno de Etnografía. II Época, 3, 99-103. La Orotava: Asociación Cultural "Día de las Tradiciones Canarias".

Trapero, M. (1999). Diccionario de toponimia canaria. Las Palmas de Gran Canaria: Universidad Nacional de Educación a Distancia. 